\title{
Generalized Superharmonic Functions with Strongly Nonlinear Operator
}

\author{
Iwona Chlebicka ${ }^{1}$ (D) . Anna Zatorska-Goldstein ${ }^{1}$ (D)
}

Received: 8 June 2020 / Accepted: 5 March 2021 / Published online: 25 March 2021

(C) The Author(s) 2021

\begin{abstract}
We study properties of $\mathcal{A}$-harmonic and $\mathcal{A}$-superharmonic functions involving an operator having generalized Orlicz growth. Our framework embraces reflexive Orlicz spaces, as well as natural variants of variable exponent and double-phase spaces. In particular, Harnack's Principle and Minimum Principle are provided for $\mathcal{A}$-superharmonic functions.
\end{abstract}

Keywords Superharmonic functions · Harnack's principle · Poisson modification · Minimum principle $\cdot$ Liouville's theorem $\cdot$ Potential theory

Mathematics Subject Classification (2010) $35 \mathrm{~J} 60 \cdot 35 \mathrm{~J} 70$

\section{Introduction}

The cornerstone of the classical potential theory is the Dirichlet problem for harmonic functions. The focus of the nonlinear potential theory is similar, however, harmonic functions are replaced by $p$-harmonic functions, that is, continuous solutions to the $p$-Laplace equation $-\Delta_{p} u=-\operatorname{div}\left(|D u|^{p-2} D u\right)=0,1<p<\infty$. There are known attempts to adapt the theory to the case when the exponent varies in space, that is $p=p(x)$ for $x \in \Omega$ or the growth is non-polynomial. Inspired by the significant attention paid lately to problems with strongly nonstandard and non-uniformly elliptic growth e.g. [11, 15, 21, 23, 30, 47, 50] we aim at developing basics of potential theory for problems with essentially broader class of operators embracing in one theory as special cases Orlicz, variable exponent and double-phase generalizations of $p$-Laplacian. To cover whole the mentioned range of general growth problems we employ the framework described in the monograph [26]. Let us stress that unlike the classical studies [34, 38] the operator we consider does not enjoy homogeneity of a form $\mathcal{A}(x, k \xi)=|k|^{p-2} k \mathcal{A}(x, \xi)$. Consequently, our class of solutions is

Iwona Chlebicka

i.chlebicka@mimuw.edu.pl

Anna Zatorska-Goldstein

azator@mimuw.edu.pl

1 Institute of Applied Mathematics and Mechanics, University of Warsaw, ul. Banacha 2, 02-097

Warsaw, Poland 
not invariant with respect to scalar multiplication. Moreover, we allow for operators whose ellipticity is allowed to vary dramatically in the space variable. What is more, we do not need to assume in the definition of $\mathcal{A}$-superharmonic function that it is integrable with some positive power, which is typically imposed in the variable exponent case, cf. e.g. [27, 44].

We study fine properties of $\mathcal{A}$-superharmonic functions defined by the Comparison Principle with respect to continuous solutions to $-\operatorname{div} \mathcal{A}(x, D u)=0$. Here $\mathcal{A}: \Omega \times \mathbb{R}^{n} \rightarrow \mathbb{R}^{n}$ is assumed to have generalized Orlicz growth expressed by the means of an inhomogeneous convex $\Phi$-function $\varphi: \Omega \times[0, \infty) \rightarrow[0, \infty)$ satisfying natural non-degeneracy and balance conditions, see Section 2 for details. In turn, the solutions belong to the Musielak-Orlicz-Sobolev space $W^{1, \varphi(\cdot)}(\Omega)$ described carefully in the monograph [26]. The assumptions on the operator are summarized below and will be referred to as (A) throughout the paper.

\subsection{Assumption (A)}

We assume that $\Omega \subset \mathbb{R}^{n}, n \geq 2$, is an open bounded set. Let a vector field $\mathcal{A}: \Omega \times \mathbb{R}^{n} \rightarrow$ $\mathbb{R}^{n}$ be a Caratheodory's function, that is $x \mapsto \mathcal{A}(x, \cdot)$ is measurable and $z \mapsto \mathcal{A}(\cdot, z)$ is continuous. Assume further that the following growth and coercivity assumptions hold true for almost all $x \in \Omega$ and all $z \in \mathbb{R}^{n} \backslash\{0\}$ :

$$
\left\{\begin{array}{l}
|\mathcal{A}(x, z)| \leq c_{1}^{\mathcal{A}} \varphi(x,|z|) /|z|, \\
c_{2}^{\mathcal{A}} \varphi(x,|z|) \leq \mathcal{A}(x, z) \cdot z
\end{array}\right.
$$

with absolute constants $c_{1}^{\mathcal{A}}, c_{2}^{\mathcal{A}}>0$ and some function $\varphi: \Omega \times[0, \infty) \rightarrow[0, \infty)$ being measurable with respect to the first variable, convex with respect to the second one and satisfying $(\mathrm{A} 0),(\mathrm{A} 1),(\mathrm{aInc})_{p}$ and $(\mathrm{aDec})_{q}$ with some $1<p \leq q<\infty$. The precise statement of these conditions is given in Section 2. We collect all parameters of the problem as data $=\operatorname{data}\left(p, q, c_{1}^{\mathcal{A}}, c_{2}^{\mathcal{A}}\right)$.

Moreover, let $\mathcal{A}$ be monotone in the sense that for a.a. $x \in \Omega$ and any distinct $z_{1}, z_{2} \in \mathbb{R}^{n}$ it holds that

$$
0<\left\langle\mathcal{A}\left(x, z_{1}\right)-\mathcal{A}\left(x, z_{2}\right), z_{1}-z_{2}\right\rangle .
$$

We shall consider weak solutions, $\mathcal{A}$-supersolutions, $\mathcal{A}$-superharmonic, and $\mathcal{A}$-harmonic functions related to the problem

$$
-\operatorname{div} \mathcal{A}(x, D u)=0 \quad \text { in } \Omega .
$$

For precise definitions see Section 3.

\subsection{Special Cases}

Besides the $p$-Laplace operator case, corresponding to the choice of $\varphi(x, s)=s^{p}, 1<p<$ $\infty$, we cover by one approach a wide range of more degenerate operators. When we take $\varphi(x, s)=s^{p(x)}$, with $p: \Omega \rightarrow \mathbb{R}$ such that $1<p_{\Omega}^{-} \leq p(x) \leq p_{\Omega}^{+}<\infty$ and satisfying logHölder condition (a special case of (A1)), we render the so-called $p(x)$-Laplace equation

$$
0=-\Delta_{p(x)} u=-\operatorname{div}\left(|D u|^{p(x)-2} D u\right) .
$$

Within the framework studied in [21] solutions to double phase version of the $p$-Laplacian

$$
0=-\operatorname{div} \mathcal{A}(x, D u)=-\operatorname{div}\left(\omega(x)\left(|D u|^{p-2}+a(x)|D u|^{q-2}\right) D u\right)
$$


are analysed with $1<p \leq q<\infty$, possibly vanishing weight $0 \leq a \in C^{0, \alpha}(\Omega)$ and $q / p \leq 1+\alpha / n$ (a special case of (A1); sharp for density of regular functions) and with a bounded, measurable, separated from zero weight $\omega$. We embrace also the borderline case between the double phase space and the variable exponent one, cf. [5]. Namely, we consider solutions to

$$
0=-\operatorname{div} \mathcal{A}(x, D u)=-\operatorname{div}\left(\omega(x)|D u|^{p-2}(1+a(x) \log (\mathrm{e}+|D u|)) D u\right)
$$

with $1<p<\infty, \log$-Hölder continuous $a$ and a bounded, measurable, separated from zero weight $\omega$. Having an $N$-function $B \in \Delta_{2} \cap \nabla_{2}$, we can allow for problems with the leading part of the operator with growth driven by $\varphi(x, s)=B(s)$ with an example of

$$
0=-\operatorname{div} \mathcal{A}(x, D u)=-\operatorname{div}\left(\omega(x) \frac{B(|D u|)}{|D u|^{2}} D u\right)
$$

with a bounded, measurable, and separated from zero weight $\omega$. To give more new examples one can consider problems stated in weighted Orlicz (if $\varphi(x, s)=a(x) B(s)$ ), variable exponent double phase (if $\varphi(x, s)=s^{p(x)}+a(x) s^{q(x)}$ ), or multi phase Orlicz cases (if $\left.\varphi(x, s)=\sum_{i} a_{i}(x) B_{i}(s)\right)$, as long as $\varphi(x, s)$ is comparable to a function doubling with respect to the second variable and it satisfies the non-degeneracy and no-jump assumptions (A0)-(A1), see Section 2.

\subsection{State of Art}

The key references for already classical nonlinear potential theory are [2, 34, 38], but its foundations date back further to $[33,35]$. A complete overview of the theory for equations with $p$-growth is presented in [42]. The first generalization of potential theory towards nonstandard growth is done in the weighted case $[48,53]$. So far significant attention was put on the variable exponent case, see e.g. [4, 27, 28, 32, 44], and analysis of related problems over metric spaces [8], there are some results obtained in the double-phase case [24], but to our best knowledge the Orlicz case is not yet covered by any comprehensive study stemming from $[45,46]$.

Let us mention the recent advances within the theory. Supersolutions to Eq. 2 are in fact solutions to measure data problems with nonnegative measure, that enjoy lately the separate interest, cf. [1, 12-14, 17, 20, 39, 42, 49] concentrating on their existence and gradient estimates. The generalization of studies on removable sets for Hölder continuous solutions provided by [40] to the case of strongly non-uniformly elliptic operators has been carried out lately in $[15,16]$. There are available various regularity results for related quasiminimizers having Orlicz or generalized Orlicz growth [25, 29, 30, 32, 36, 37, 46]. For other recent developments in the understanding of the functional setting we refer also to [3, 19, 22, 31].

\subsection{Applications}

This kind of results are useful in getting potential estimates for solutions to measure data problems, entailing further regularity properties of their solutions, cf. [38, 42, 43]. Particularly, the Maximum and Minimum Principles for $p$-harmonic functions together with properties of Poisson modification of $p$-superharmonic functions are important tools in getting Wolff potential estimates via the methods of [41, 52]. In fact, developing this approach further, we employ the results of our paper in the proof of Wolff potential estimates for problems with Orlicz growth [18]. They directly entail many natural and sharp regularity 
consequences and Orlicz version of the Hedberg-Wolff Theorem yielding full characterization of the natural dual space to the space of solutions by the means of the Wolff potential (see [35] for the classical version).

\subsection{Results and Organization}

Section 2 is devoted to notation and basic information on the setting. In Section 3 we define weak solutions, $\mathcal{A}$-supersolutions, $\mathcal{A}$-harmonic and $\mathcal{A}$-superharmonic functions and provide proofs of their fundamental properties including the Harnack inequality for $\mathcal{A}$-harmonic functions (Theorem 1). Further analysis of $\mathcal{A}$-superharmonic functions is carried out in Section 4. Let us stress that due to the lack of the homogeneity of the operator this part substantially differs from the classical references $[34,38]$ as well as $[28,32]$. We prove there Harnack's Principle (Theorem 2), fundamental properties of Poisson's modification (Theorem 3), and Strong Minimum Principle (Theorem 4) together with their consequence of the inequality of the Harnack type (Theorem 5) for $\mathcal{A}$-harmonic functions. We point out that the proof of Strong Minimum Principle strongly relies on the control of the size of the balls in the terms of gradients of truncations of $\mathcal{A}$-superharmonic function, which is uniform with respect to the truncation level, see Remark 4.13 .

\section{Preliminaries}

\subsection{Notation}

In the following we shall adopt the customary convention of denoting by $c$ a constant that may vary from line to line. Sometimes to skip rewriting a constant, we use $\lesssim$. By $a \simeq b$, we mean $a \lesssim b$ and $b \lesssim a$. By $B_{R}$ we shall denote a ball usually skipping prescribing its center, when it is not important. Then by $c B_{R}=B_{c R}$ we mean a ball with the same center as $B_{R}$, but with rescaled radius $c R$. With $U \subset \mathbb{R}^{n}$ being a measurable set with finite and positive measure $|U|>0$, and with $f: U \rightarrow \mathbb{R}^{k}, k \geq 1$ being a measurable map, by

$$
f_{U} f(x) d x=\frac{1}{|U|} \int_{U} f(x) d x
$$

we mean the integral average of $f$ over $U$. We make use of symmetric truncation on level $k>0, T_{k}: \mathbb{R} \rightarrow \mathbb{R}$, defined as follows

$$
T_{k}(s)= \begin{cases}s & |s| \leq k, \\ k \frac{s}{|s|} & |s| \geq k .\end{cases}
$$

\subsection{Generalized Orlicz Functions}

We employ the formalism introduced in the monograph [26]. Let us present the framework.

For $L \geq 1$ a real-valued function $f$ is $L$-almost increasing, if $L f(s) \geq f(t)$ for $s>t ; f$ is called $L$-almost decreasing if $L f(s) \leq f(t)$ for $s>t$.

Definition 2.1 We say that $\varphi: \Omega \times[0, \infty) \rightarrow[0, \infty]$ is a convex $\Phi$-function, and write $\varphi \in \Phi_{c}(\Omega)$, if the following conditions hold:

(i) For every $s \in[0, \infty)$ the function $x \mapsto \varphi(x, s)$ is measurable and for a.e. $x \in \Omega$ the function $s \mapsto \varphi(x, s)$ is increasing, convex, and left-continuous. 
(ii) $\varphi(x, 0)=\lim _{s \rightarrow 0^{+}} \varphi(x, s)=0$ and $\lim _{s \rightarrow \infty} \varphi(x, s)=\infty$ for a.e. $x \in \Omega$.

Further, we say that $\varphi \in \Phi_{c}(\Omega)$ satisfies

(aInc) $p \quad$ if there exist $L \geq 1$ and $p>1$ such that $s \mapsto \varphi(x, s) / s^{p}$ is $L$-almost increasing in $[0, \infty)$ for every $x \in \Omega$,

$(\mathrm{aDec})_{q} \quad$ if there exist $L \geq 1$ and $q>1$ such that $s \mapsto \varphi(x, s) / s^{q}$ is $L$-almost decreasing in $[0, \infty)$ for every $x \in \Omega$.

By $\varphi^{-1}$ we denote the inverse of a convex $\Phi$-function $\varphi$ with respect to the second variable, that is

$$
\varphi^{-1}(x, \tau):=\inf \{s \geq 0: \varphi(x, s) \geq \tau\} .
$$

We shall consider those $\varphi \in \Phi_{c}(\Omega)$, which satisfy the following set of conditions.

(A0) There exists $\beta_{0} \in(0,1]$ such that $\varphi\left(x, \beta_{0}\right) \leq 1$ and $\varphi\left(x, 1 / \beta_{0}\right) \geq 1$ for all $x \in \Omega$.

(A1) There exists $\beta_{1} \in(0,1)$, such that for every ball $B$ with $|B| \leq 1$ it holds that

$$
\beta_{1} \varphi^{-1}(x, s) \leq \varphi^{-1}(y, s) \text { for every } s \in[1,1 /|B|] \text { and a.e. } x, y \in B \cap \Omega \text {. }
$$

(A2) For every $s>0$ there exist $\beta_{2} \in(0,1]$ and $h \in L^{1}(\Omega) \cap L^{\infty}(\Omega)$, such that

$$
\varphi\left(x, \beta_{2} r\right) \leq \varphi(y, r)+h(x)+h(y) \quad \text { for a.e. } x, y \in \Omega \text { whenever } \varphi(y, r) \in[0, s] .
$$

Condition (A0) is imposed in order to exclude degeneracy, while (A1) can be interpreted as local continuity. Fundamental role is played also by (A2) which imposes balance of the growth of $\varphi$ with respect to its variables separately.

The Young conjugate of $\varphi \in \Phi_{c}(\Omega)$ is the function $\tilde{\varphi}: \Omega \times[0, \infty) \rightarrow[0, \infty]$ defined as $\tilde{\varphi}(\underset{\varphi}{x}, s)=\sup \{r \cdot s-\varphi(x, r): r \in[0, \infty)\}$. Note that Young conjugation is involute, i.e. $(\widetilde{\widetilde{\varphi}})=\varphi$. Moreover, if $\varphi \in \Phi_{c}(\Omega)$, then $\widetilde{\varphi} \in \Phi_{c}(\Omega)$. For $\varphi \in \Phi_{c}(\Omega)$, the following inequality of Fenchel-Young type holds true

$$
r s \leq \varphi(x, r)+\tilde{\varphi}(x, s) .
$$

We say that a function $\varphi$ satisfies $\Delta_{2}$-condition (and write $\varphi \in \Delta_{2}$ ) if there exists a constant $c>0$, such that for every $s \geq 0$ it holds $\varphi(x, 2 s) \leq c(\varphi(x, s)+1)$. If $\tilde{\varphi} \in \Delta_{2}$, we say that $\varphi$ satisfies $\nabla_{2}$-condition and denote it by $\varphi \in \nabla_{2}$. If $\varphi, \widetilde{\varphi} \in \Delta_{2}$, then we call $\varphi$ a doubling function. If $\varphi \in \Phi_{c}(\Omega)$ satisfies $(\mathrm{aInc})_{p}$ and $(\mathrm{aDec})_{q}$, then $\varphi \simeq \psi_{1}$ with some $\psi_{1} \in \Phi_{c}(\Omega)$ satisfying $\Delta_{2}$-condition and $\tilde{\varphi} \simeq \widetilde{\psi}_{2}$ with some $\widetilde{\psi}_{2} \in \Phi_{c}(\Omega)$ satisfying $\Delta_{2-}$ condition, so we can assume that functions within our framework are doubling. Note that also $\psi_{1} \simeq \widetilde{\psi}_{2}$.

In fact, within our framework

$$
\widetilde{\varphi}(x, \varphi(x, s) / s) \sim \varphi(x, s) \text { for a.e. } x \in \Omega \text { and all } s>0
$$

for some constants depending only on $p$ and $q$.

\subsection{Function Spaces}

For a comprehensive study of these spaces we refer to [26]. We always deal with spaces generated by $\varphi \in \Phi_{c}(\Omega)$ satisfying (aInc) ${ }_{p},(\mathrm{aDec})_{q},(\mathrm{~A} 0),(\mathrm{A} 1)$, and (A2). For $f \in L^{0}(\Omega)$ we define the modular $\varrho_{\varphi(\cdot), \Omega}$ by

$$
\varrho_{\varphi(\cdot), \Omega}(f)=\int_{\Omega} \varphi(x,|f(x)|) d x .
$$

When it is clear from the context we omit assigning the domain. 
The Musielak-Orlicz space is defined as the set

$$
L^{\varphi(\cdot)}(\Omega)=\left\{f \in L^{0}(\Omega): \lim _{\lambda \rightarrow 0^{+}} \varrho_{\varphi(\cdot), \Omega}(\lambda f)=0\right\}
$$

endowed with the Luxemburg norm

$$
\|f\|_{\varphi(\cdot)}=\inf \left\{\lambda>0: \varrho_{\varphi(\cdot), \Omega}\left(\frac{1}{\lambda} f\right) \leq 1\right\} .
$$

For $\varphi \in \Phi_{c}(\Omega)$, the space $L^{\varphi(\cdot)}(\Omega)$ is a Banach space [26, Theorem 2.3.13]. Moreover, the following Hölder inequality holds true

$$
\|f g\|_{L^{1}(\Omega)} \leq 2\|f\|_{L^{\varphi(\cdot)}(\Omega)}\|g\|_{L^{\widetilde{\varphi}(\cdot)}(\Omega)} .
$$

We define the Musielak-Orlicz-Sobolev space $W^{1, \varphi(\cdot)}(\Omega)$ as follows

$$
W^{1, \varphi(\cdot)}(\Omega)=\left\{f \in W_{l o c}^{1,1}(\Omega): \quad f,|D f| \in L^{\varphi(\cdot)}(\Omega)\right\},
$$

where $D$ stands for distributional derivative. The space is considered endowed with the norm

$$
\|f\|_{W^{1, \varphi(\cdot)}(\Omega)}=\inf \left\{\lambda>0: \varrho_{\varphi(\cdot), \Omega}\left(\frac{1}{\lambda} f\right)+\varrho_{\varphi(\cdot), \Omega}\left(\frac{1}{\lambda} D f\right) \leq 1\right\} .
$$

By $W_{0}^{1, \varphi(\cdot)}(\Omega)$ we denote a closure of $C_{0}^{\infty}(\Omega)$ under the above norm.

Because of the growth conditions $W^{1, \varphi(\cdot)}(\Omega)$ is a separable and reflexive space. Moreover, smooth functions are dense there.

Remark 2.2 [26] If $\varphi \in \Phi_{c}(\Omega)$ satisfies $(\mathrm{aInc})_{p},(\mathrm{aDec})_{q},(\mathrm{~A} 0),(\mathrm{A} 1),(\mathrm{A} 2)$, then strong (norm) topology of $W^{1, \varphi(\cdot)}(\Omega)$ coincides with the sequensional modular topology. Moreover, smooth functions are dense in this space in both topologies.

Note that as a consequence of [6, Lemma 2.1] for every function $u$, such that $T_{k}(u) \in$ $W^{1, \varphi(\cdot)}(\Omega)$ for every $k>0$ (with $T_{k}$ given by Eq. 2.1) there exists a (unique) measurable function $Z_{u}: \Omega \rightarrow \mathbb{R}^{n}$ such that

$$
D T_{k}(u)=\chi_{\{|u|<k\}} Z_{u} \quad \text { for a.e. in } \Omega \text { and for every } k>0 .
$$

With an abuse of notation, we denote $Z_{u}$ simply by $D u$ and call it a generalized gradient.

\subsection{The Operator}

Let us motivate that the growth and coercivity conditions from Eq. 1 imply the expected proper definition of the operator involved in problem (2). We notice that in our regime the operator $\mathfrak{A}_{\varphi(\cdot)}$ defined as

$$
\mathfrak{A}_{\varphi(\cdot)} v:=\mathcal{A}(x, D v)
$$

is well defined as $\mathfrak{A}_{\varphi(\cdot)}: W_{0}^{1, \varphi(\cdot)}(\Omega) \rightarrow\left(W_{0}^{1, \varphi(\cdot)}(\Omega)\right)^{\prime}$ via

$$
\left\langle\mathfrak{A}_{\varphi(\cdot)} v, w\right\rangle:=\int_{\Omega} \mathcal{A}(x, D v) \cdot D w d x \quad \text { for } \quad w \in C_{0}^{\infty}(\Omega),
$$

where $\langle\cdot, \cdot\rangle$ denotes dual pairing between reflexive Banach spaces $W_{0}^{1, \varphi(\cdot)}(\Omega)$ and $W_{0}^{1, \varphi(\cdot)}(\Omega)^{\prime}$. Indeed, when $v \in W_{0}^{1, \varphi(\cdot)}(\Omega)$ and $w \in C_{0}^{\infty}(\Omega)$, growth conditions (1), Hölder's inequality (5), equivalence (3), and Poincaré inequality [26, Theorem 6.2.8] justify 
that

$$
\begin{aligned}
\left|\left\langle\mathfrak{A}_{\varphi(\cdot)} v, w\right\rangle\right| & \leq c \int_{\Omega} \frac{\varphi(x,|D v|)}{|D v|}|D w| d x \leq c\left\|\frac{\varphi(\cdot,|D v|)}{|D v|}\right\|_{L^{\widetilde{\varphi}(\cdot)}(\Omega)}\|D w\|_{L^{\varphi(\cdot)}(\Omega)} \\
& \leq c\|D v\|_{L^{\varphi(\cdot)}(\Omega)}\|D w\|_{L^{\varphi(\cdot)}(\Omega)} \leq c\|w\|_{W_{0}^{1, \varphi(\cdot)}(\Omega)} .
\end{aligned}
$$

By density argument, the operator is well-defined on $W_{0}^{1, \varphi(\cdot)}(\Omega)$.

\section{Various Types of Solutions and the Notion of $\mathcal{A}$-Harmonicity}

All the problems are considered under Assumption (A).

\subsection{Definitions and Basic Remarks}

A continuous function $u \in W_{l o c}^{1, \varphi(\cdot)}(\Omega)$ is called an $\mathcal{A}$-harmonic function in an open set $\Omega$ if it is a (weak) solution to the equation $-\operatorname{div} \mathcal{A}(x, D u)=0$, i.e.,

$$
\int_{\Omega} \mathcal{A}(x, D u) \cdot D \phi d x=0 \text { for all } \phi \in C_{0}^{\infty}(\Omega)
$$

Existence and uniqueness of $\mathcal{A}$-harmonic functions are proven in [16].

Proposition 3.1 Under Assumption (A) if $\Omega$ is bounded and $w \in W^{1, \varphi(\cdot)}(\Omega) \cap C(\Omega)$, then there exists a unique solution $u \in W^{1, \varphi(\cdot)}(\Omega) \cap C(\Omega)$ to problem

$$
\left\{\begin{array}{l}
-\operatorname{div} \mathcal{A}(x, D u)=0 \quad \text { in } \Omega, \\
u-w \in W_{0}^{1, \varphi(\cdot)}(\Omega) .
\end{array}\right.
$$

Moreover, $u$ is locally bounded and for every $E \Subset \Omega$ we have

$$
\|u\|_{L^{\infty}(E)} \leq c\left(\operatorname{data},\|D u\|_{W^{1, \varphi(\cdot)}(\Omega)}\right) .
$$

We call a function $u \in W_{l o c}^{1, \varphi(\cdot)}(\Omega)$ a (weak) $\mathcal{A}$-supersolution to Eq. 8 if $-\operatorname{div} \mathcal{A}(x, D u) \geq$ 0 weakly in $\Omega$, that is

$$
\int_{\Omega} \mathcal{A}(x, D u) \cdot D \phi d x \geq 0 \text { for all } 0 \leq \phi \in C_{0}^{\infty}(\Omega)
$$

and a (weak) $\mathcal{A}$-subsolution if $-\operatorname{div} \mathcal{A}(x, D u) \leq 0$ weakly in $\Omega$, that is

$$
\int_{\Omega} \mathcal{A}(x, D u) \cdot D \phi d x \leq 0 \text { for all } 0 \leq \phi \in C_{0}^{\infty}(\Omega) .
$$

By density of smooth functions we can use actually test functions from $W_{0}^{1, \varphi(\cdot)}(\Omega)$.

The classes of $\mathcal{A}$-superharmonic and $\mathcal{A}$-subharmonic are defined by the Comparison Principle.

Definition 3.2 We say that function $u$ is $\mathcal{A}$-superharmonic if

(i) $u$ is lower semicontinuous;

(ii) $u \neq \equiv$ in any component of $\Omega$;

(iii) for any $K \Subset \Omega$ and any $\mathcal{A}$-harmonic $h \in C(\bar{K})$ in $K, u \geq h$ on $\partial K$ implies $u \geq h$ in $K$. 
We say that an upper semicontinuous function $u$ is $\mathcal{A}$-subharmonic if $(-u)$ is $\mathcal{A}$ superharmonic.

The above definitions have the following direct consequences.

Lemma 3.3 An $\mathcal{A}$-superharmonic function $u$ is locally bounded from below. An $\mathcal{A}$-subharmonic function $u$ is locally bounded from above.

Lemma 3.4 If $u$ is $\mathcal{A}$-harmonic, then it is $\mathcal{A}$-supersolution, $\mathcal{A}$-subsolution, $\mathcal{A}$ superharmonic, and $\mathcal{A}$-subharmonic.

By minor modification of the proof of [36, Lemma 4.3] we get the following fact.

Lemma 3.5 Let $u \in W^{1, \varphi(\cdot)}(\Omega)$ be an $\mathcal{A}$-supersolution to Eq. 8, and $v \in W^{1, \varphi(\cdot)}(\Omega)$ be an $\mathcal{A}$-subsolution to Eq. 8. If $\min (u-v) \in W_{0}^{1, \varphi(\cdot)}(\Omega)$, then $u \geq v$ a.e. in $\Omega$.

We have the following estimate for $\mathcal{A}$-supersolutions.

Lemma 3.6 (Lemma 5.1, [16]) If $u \in W^{1, \varphi(\cdot)}(\Omega)$ is a nonnegative $\mathcal{A}$-supersolution, $B \Subset$ $\Omega$, and $\eta \in C_{0}^{1}(B)$ is such that $0 \leq \eta \leq 1$. Then for all $\gamma \in(1, p)$ there holds

$$
\int_{B} u^{-\gamma} \eta^{q} \varphi(x,|D u|) d x \leq c \int_{B} u^{-\gamma} \varphi(x,|D \eta| u) d x \quad \text { with } c=c(\operatorname{data}, \gamma) .
$$

It is well known that solutions, subsolutions, and supersolutions can be described by the theory of quasiminimizers. Since many of the results on quasiminizers from [25] apply to our $\mathcal{A}$-harmonic functions we shall recall the definition.

Among all functions having the same 'boundary datum' $w \in W^{1, \varphi(\cdot)}(\Omega)$ the function $u \in W^{1, \varphi(\cdot)}$ is a quasiminimizer if it has the least energy up to a factor $C$, that is if $(u-w) \in$ $W_{0}^{1, \varphi(\cdot)}(\Omega)$ and

$$
\int_{\Omega} \varphi(x,|D u|) d x \leq C \int_{\Omega} \varphi(x,|D(u+v)|) d x
$$

holds true with an absolute constant $C>0$ for every $v \in W_{0}^{1, \varphi(\cdot)}(\Omega)$. We call a function $u$ superquasiminimizer (subquasiminimizer) if Eq. 9 holds for all $v$ as above that are additionally nonnegative (nonpositive).

Lemma 3.7 An $\mathcal{A}$-harmonic function u is a quasiminimizer.

Proof Let us take an arbitrary $v \in W_{0}^{1, \varphi(\cdot)}(\Omega)$. We may write $v=w+\tilde{v}-u$ with 'boundary datum' $w$ and any $\tilde{v} \in W_{0}^{1, \varphi(\cdot)}(\Omega)$, and upon testing the Eq. 2 with $v$ we obtain

$$
\int_{\Omega} \mathcal{A}(x, D u) \cdot D u d x=\int_{\Omega} \mathcal{A}(x, D u) \cdot D(w+\tilde{v}) d x .
$$


Then by coercivity of $\mathcal{A}$, Young's inequality, growth of $\mathcal{A}$ and doubling growth of $\varphi$, for every $\varepsilon>0$ we have

$$
\begin{aligned}
c_{2}^{\mathcal{A}} \int_{\Omega} \varphi(x,|D u|) d x & \leq \int_{\Omega} \mathcal{A}(x, D u) \cdot D u d x=\int_{\Omega} \mathcal{A}(x, D u) \cdot D(w+\tilde{v}) d x \\
& \leq \varepsilon \int_{\Omega} \widetilde{\varphi}(x,|\mathcal{A}(x, D u)|) d x+c(\varepsilon) \int_{\Omega} \varphi(x,|D(w+\tilde{v})|) d x \\
& \leq \varepsilon \int_{\Omega} \widetilde{\varphi}\left(x, c_{1}^{\mathcal{A}} \varphi(x,|D u|) /|D u|\right) d x+c(\varepsilon) \int_{\Omega} \varphi(x,|D(w+\tilde{v})|) d x \\
& \leq \varepsilon \bar{c} \int_{\Omega} \varphi(x,|D u|) d x+c(\varepsilon) \int_{\Omega} \varphi(x,|D(w+\tilde{v})|) d x
\end{aligned}
$$

with $\bar{c}=\bar{c}($ data $)>0$. Let us choose $\varepsilon>0$ small enough for the first term on the righthand side can be absorbed on the left-hand side. By rearranging terms, and using the fact that $u+v=w+\tilde{v}$ we get that

$$
\int_{\Omega} \varphi(x,|D u|) d x \leq C \int_{\Omega} \varphi(x,|D(u+v)|) d x \quad \text { with } C=C(\operatorname{data})>0 .
$$

Hence we get the claim.

By the same calculations as in the above proof we have the following corollary.

Corollary 3.8 If $u$ is $\mathcal{A}$-supersolution, then $u$ is a superquasiminizer, i.e. Eq. 9 holds for all nonnegative $v \in W_{0}^{1, \varphi(\cdot)}(\Omega)$.

\subsection{Obstacle Problem}

We consider the set

$$
\mathcal{K}_{\psi, w}(\Omega):=\left\{v \in W^{1, \varphi(\cdot)}(\Omega): v \geq \psi \text { a.e. in } \Omega \text { and } v-w \in W_{0}^{1, \varphi(\cdot)}(\Omega)\right\},
$$

where we call $\psi: \Omega \rightarrow \bar{R}$ the obstacle and $w \in W^{1, \varphi(\cdot)}(\Omega)$ the boundary datum. If $\mathcal{K}_{\psi, w}(\Omega) \neq \varnothing$ by a solution to the obstacle problem we mean a function $u \in \mathcal{K}_{\psi, w}(\Omega)$ satisfying

$$
\int_{\Omega} \mathcal{A}(x, D u) \cdot D(v-u) d x \geq 0 \text { for all } v \in \mathcal{K}_{\psi, w}(\Omega) .
$$

We note the following basic information on the existence, the uniqueness, and the Comparison Principle for the obstacle problem are provided in [37] and [16, Section 4].

Proposition 3.9 (Theorem 2, [16]) Under Assumption(A) let the obstacle $\psi \in$ $W^{1, \varphi(\cdot)}(\Omega) \cup\{-\infty\}$ and the boundary datum $w \in W^{1, \varphi(\cdot)}(\Omega)$ be such that $\mathcal{K}_{\psi, w}(\Omega) \neq \emptyset$. Then there exists a function $u \in \mathcal{K}_{\psi, w}(\Omega)$ being a unique solution to the $\mathcal{K}_{\psi, w}(\Omega)$-obstacle problem (11). Moreover, if $\psi \in W^{1, \varphi(\cdot)}(\Omega) \cap C(\Omega)$, then $v$ is continuous and is $\mathcal{A}$-harmonic in the open set $\{x \in \Omega: u(x)>\psi(x)\}$.

For more properties of solutions to related obstacle problems see also [9, 10, 15, 27, 36, 51]. In particular, in [36] several basic properties of quasiminimizers to related variational obstacle problem are proven. 
Proposition 3.10 (Proposition 4.3, [16]) Let $B_{r} \Subset B_{R} \subset \Omega$. Under assumptions of Proposition 3.9,

(1) if $u$ is a solution to the $\mathcal{K}_{\psi, w}(\Omega)$-obstacle problem (11), then there exists $c=$ $c($ data, $n)$, such that

$$
\int_{B_{R}} \varphi\left(x,\left|D(u-k)_{+}\right|\right) d x \leq c \int_{B_{R}} \varphi\left(x, \frac{(u-k)_{+}}{R-r}\right) d x \text {, where } k \geq \sup _{x \in B_{R}} \psi(x) .
$$

(2) if $u$ is a $\mathcal{A}$-supersolution to Eq. 8 in $\Omega$, then there exists $c=c($ data, $n)$, such that

$$
\int_{B_{R}} \varphi\left(x,\left|D u_{-}\right|\right) d x \leq c \int_{B_{R}} \varphi\left(x, \frac{\left|u_{-}\right|}{R}\right) d x .
$$

Note that in fact in [36, Proposition 4.3] only (1) is proven in detail, but (2) follows by the same arguments.

\section{$4 \mathcal{A}$-Superharmonic Functions}

\subsection{Basic Observations}

Proposition 4.1 (Comparison Principle) Suppose $u$ is $\mathcal{A}$-superharmonic and $v$ is $\mathcal{A}$ subharmonic in $\Omega$. If $\limsup _{y \rightarrow x} v(y) \leq \liminf _{y \rightarrow x} u(y)$ for all $x \in \partial \Omega$ (excluding the cases $-\infty \leq-\infty$ and $\infty \leq \infty)$, then $v \leq u$ in $\Omega$.

Proof When we fix $x \in \Omega$ and $\varepsilon>0$, by the assumption we can find a regular open set $D \Subset \Omega$, such that $v<u+\varepsilon$ on $\partial D$. Pick a decreasing sequence $\left\{\phi_{k}\right\} \subset C^{\infty}(\Omega)$ converging to $v$ pointwise in $\bar{D}$. Since $\partial D$ is compact by lower semicontinuity of $(u+\varepsilon)$ we infer that $\phi_{k} \leq u+\varepsilon$ on $\partial D$ for some $k$. We take a function $h$ being $\mathcal{A}$-harmonic in $D$ coinciding with $\phi_{k}$ on $\partial D$. By definition it is continuous up to a boundary of $D$. Therefore, $v \leq h \leq u+\varepsilon$ on $\partial D$ and so $v \leq h \leq u+\varepsilon$ in $D$ as well. We get the claim by letting $\varepsilon \rightarrow 0$.

Corollary 4.2 Having the Comparison Principle one can deduce what follows.

(i) If $a \in \mathbb{R}$ and $u$ is $\mathcal{A}$-superharmonic in $\Omega$, then so is $u+a$.

(ii) If $u$ and $v$ are $\mathcal{A}$-superharmonic in $\Omega$, then so is $\min \{u, v\}$.

(iii) Suppose $u$ is not identically $\infty$, then $u$ is $\mathcal{A}$-superharmonic in $\Omega$ if and only if $\min \{u, k\}$ is $\mathcal{A}$-superharmonic in $\Omega$ for every $k=1,2, \ldots$

(iv) The function $u$ is $\mathcal{A}$-superharmonic in $\Omega$, if it is $\mathcal{A}$-superharmonic in every component of $\Omega$.

(v) If $u$ is $\mathcal{A}$-superharmonic and finite a.e. in $\Omega$ and $E \subset \Omega$ is a nonempty open subset, then $u$ is $\mathcal{A}$-superharmonic in $E$.

Lemma 4.3 Suppose $D \subset \Omega$, $u$ is $\mathcal{A}$-superharmonic in $\Omega$, and $v$ is $\mathcal{A}$-superharmonic in D. If the function

$$
w= \begin{cases}\min \{u, v\} & \text { in } D, \\ u & \text { in } \Omega \backslash D\end{cases}
$$

is lower semicontinuous, then it is $\mathcal{A}$-superharmonic in $\Omega$. 
Proof Let $E \Subset \Omega$ be open and $h$ be an $\mathcal{A}$-harmonic function, such that $h \leq w$ on $\partial E$. By the Comparison Principle of Proposition 4.1 we infer that $h \leq w$ in $\bar{E}$. Since $w$ is lower semicontinuous, for every $x \in \partial D \cap E$ it holds that

$$
\lim _{\substack{y \in D \cap \Omega \\ y \rightarrow x}} h(y) \leq u(x)=w(x) \leq \liminf _{\substack{y \in D \cap \Omega \\ y \rightarrow x}} v(y) .
$$

Consequently, for every $x \in \partial(D \cap E)$ one has

$$
\lim _{\substack{y \in D \cap \Omega \\ y \rightarrow x}} h(y) \leq w(x) \leq \liminf _{\substack{y \in D \cap \Omega \\ y \rightarrow x}} w(y) .
$$

By the Comparison Principle of Proposition 4.1 also $h \leq w$ in $D \cap E$. Then $h \leq w$ in $E$, what was to prove.

Lemma 4.4 If $u$ is a continuous $\mathcal{A}$-supersolution, then it is $\mathcal{A}$-superharmonic.

Proof Since $u$ is continuous and finite a.e. (because it belongs to $W_{l o c}^{1, \varphi(\cdot)}(\Omega)$ ), we have to prove only that Comparison Principle for $\mathcal{A}$-superharmonic functions holds.

Let $G \Subset \Omega$ be an open set, and let $h$ be a continuous, $\mathcal{A}$-harmonic function in $G$, such that $h \leq u$ on $\partial G$. Fix $\epsilon>0$ and choose and open set $E \Subset G$ such that $u+\epsilon \geq h$ in $G \backslash E$. Since the function $\min \{u+\epsilon-h, 0\}$ has compact support, it belongs to $W^{1, \varphi(\cdot)}(E)$. Hence Lemma 3.5 implies $u+\epsilon \geq h$ in $E$, and therefore a.e. in $G$. Since the function is continuous, the inequality is true in each point of $G$. As $\epsilon$ was chosen arbitrary, the claim follows.

We shall prove that $\mathcal{A}$-superharmonic functions can be approximated from below by $\mathcal{A}$-supersolutions.

Proposition 4.5 Let $u$ be $\mathcal{A}$-superharmonic in $\Omega$ and let $G \Subset \Omega$. Then there exists a nondecreasing sequence of continuous $\mathcal{A}$-supersolutions $\left\{u_{j}\right\}$ in $G$ such that $u=\lim _{j \rightarrow \infty} u_{j}$ pointwise in $G$. For nonnegative $u$, approximate functions $u_{j}$ can be chosen nonnegative as well.

Proof Since $u$ is lower semicontinuous in $\bar{G}$, it is bounded from below and there exists a nondecreasing sequence $\left\{\phi_{j}\right\}$ of Lipschitz functions on $\bar{G}$ such that $u=\lim _{j \rightarrow \infty} \phi_{j}$ in $G$. For nonnegative $u$, obviously $\phi_{j}, j \in \mathbb{N}$ can be chosen nonnegative as well. Let $u_{j}$ be the solution of the $\mathcal{K}_{\phi_{j}, \phi_{j}}(G)$-obstacle problem which by Proposition 3.9 is continuous and

$$
\phi_{j}<u_{j} \quad \text { in the open set } A_{j}=\left\{x \in G: \phi_{j} \neq u_{j}\right\} .
$$

Moreover, $u_{j}$ is $\mathcal{A}$-harmonic in $A_{j}$. By Comparison Principle from Proposition 4.1 we infer that the sequence $\left\{u_{j}\right\}$ is nondecreasing. Since $u$ is $\mathcal{A}$-superharmonic, we have $u_{j} \leq u$ in $A_{j}$. Then consequently $\phi_{j} \leq u_{j} \leq u$ in $G$. Passing to the limit with $j \rightarrow \infty$ we get that $u=\lim _{j \rightarrow \infty} u_{j}$, what completes the proof.

Lemma 4.6 If $u$ is $\mathcal{A}$-superharmonic in $\Omega$ and locally bounded from above, then $u \in$ $W_{\text {loc }}^{1, \varphi(\cdot)}(\Omega)$ and $u$ is $\mathcal{A}$-supersolution in $\Omega$.

Proof Fix open sets $E \Subset G \Subset \Omega$. By Proposition 4.5 there exists a nondecreasing sequence of continuous $\mathcal{A}$-supersolutions $\left\{u_{j}\right\}$ in $G$ such that $u=\lim _{j \rightarrow \infty} u_{j}$ pointwise in $G$. Since $u$ is locally bounded we may assume $u_{j} \leq u<0$ in $G$. It follows from Proposition 3.10 
that the sequence $\left\{\left|D u_{j}\right|\right\}$ is locally bounded in $L^{\varphi(\cdot)}(G)$. Since $u_{j} \rightarrow u$ a.e. in $G$, it follows that $u \in W^{1, \varphi(\cdot)}(G)$, and $D u_{j} \rightarrow D u$ weakly in $L^{\varphi(\cdot)}(G)$.

We need to show now that $u$ is an $\mathcal{A}$-supersolution in $\Omega$. To this end we first prove that (up to a subsequence) gradients $\left\{D u_{j}\right\}$ converge a.e. in $G$. We start with proving that

$$
I_{j}=\int_{E}\left(\mathcal{A}(x, D u)-\mathcal{A}\left(x, D u_{j}\right)\right) \cdot\left(D u-D u_{j}\right) d x \rightarrow 0 \quad \text { as } j \rightarrow \infty .
$$

Choose $\eta \in C_{0}^{\infty}(G)$ such that $0 \leq \eta \leq 1$, and $\eta=1$ in $E$. Using $\psi=\eta\left(u-u_{j}\right)$ as a test function for the $\mathcal{A}$-supersolution $u_{j}$ and applying the Hölder inequality, the doubling property of $\varphi$, and the Lebesgue dominated monotone convergence theorem we obtain

$$
\begin{aligned}
-\int_{G} \eta \mathcal{A}\left(x, D u_{j}\right) & \cdot\left(D u-D u_{j}\right) d x \leq \int_{G}\left(u-u_{j}\right) \mathcal{A}\left(x, D u_{j}\right) \cdot D \eta d x \\
\leq & 2\left\|\left(u-u_{j}\right) D \eta\right\|_{L^{\varphi(\cdot)}(G)}\left\|\mathcal{A}\left(\cdot, D u_{j}\right)\right\|_{L^{\widetilde{\varphi}(\cdot)}(G)} \\
\leq & c\left\|u-u_{j}\right\|_{L^{\varphi(\cdot)}(G)} \rightarrow 0 .
\end{aligned}
$$

Moreover, since

$$
\eta \mathcal{A}(\cdot, D u) \in L^{\widetilde{\varphi}(\cdot)}(G),
$$

the weak convergence $D u_{j} \rightarrow D u$ in $L^{\varphi(\cdot)}(G)$ implies

$$
\int_{G} \eta \mathcal{A}(x, D u) \cdot\left(D u-D u_{j}\right) d x \rightarrow 0 .
$$

Then, since $\eta\left(\mathcal{A}(x, D u)-\mathcal{A}\left(x, D u_{j}\right)\right) \cdot\left(D u-D u_{j}\right) \geq 0$ a.e. in $G$, we conclude with Eq. 12 . Since the integrand in $I_{j}$ is nonnegative, we may pick up a subsequence (still denoted $u_{j}$ ) such that

$$
\left(\mathcal{A}(x, D u(x))-\mathcal{A}\left(x, D u_{j}(x)\right)\right) \cdot\left(D u(x)-D u_{j}(x)\right) \rightarrow 0 \text { for a.a. } x \in E .
$$

Fix $x \in E$ such that Eq. 13 is valid, and that $|D u(x)|<\infty$. Upon choosing further subsequence we may assume that

$$
D u_{j}(x) \rightarrow \xi \in \overline{\mathbb{R}^{n}}
$$

Since we have

$$
\begin{aligned}
(\mathcal{A}(x, D u(x))- & \left.\mathcal{A}\left(x, D u_{j}(x)\right)\right) \cdot\left(D u(x)-D u_{j}(x)\right) \\
& \geq c_{2}^{\mathcal{A}} \varphi\left(x,\left|D u_{j}(x)\right|\right)-c_{1}^{\mathcal{A}} \frac{\varphi(x,|D u(x)|)}{|D u(x)|}\left|D u_{j}(x)\right|-c_{1}^{\mathcal{A}} \frac{\varphi\left(x,\left|D u_{j}(x)\right|\right)}{\left|D u_{j}(x)\right|}|D u(x)| \\
& \geq c(\operatorname{data},|D u(x)|) \varphi\left(x,\left|D u_{j}(x)\right|\right)\left(1-\frac{\left|D u_{j}(x)\right|}{\varphi\left(x,\left|D u_{j}(x)\right|\right)}-\frac{1}{\left|D u_{j}(x)\right|}\right)
\end{aligned}
$$

and Eq. 13 is true, it must follow that $|\xi|<\infty$.

Since the mapping $\zeta \mapsto \mathcal{A}(x, \zeta)$ is continuous, we have

$$
(\mathcal{A}(x, D u(x))-\mathcal{A}(x, \xi)) \cdot(D u(x)-\xi)=0
$$

and it follows that $\xi=D u(x)$, and

$$
D u_{j}(x) \rightarrow D u(x) \quad \text { for a.e. } x \in E,
$$

and

$$
\mathcal{A}\left(\cdot, D u_{j}\right) \rightarrow \mathcal{A}(\cdot, D u) \quad \text { weakly in } L^{\widetilde{\varphi}(\cdot)} .
$$


Therefore that $u$ is an $\mathcal{A}$-supersolution of Eq. 8. Indeed, if $\phi \in C_{0}^{\infty}(\Omega), \phi \geq 0$ is such that $\operatorname{supp} \phi \subset E$, then $D \phi \in L^{\varphi(\cdot)}(E)$ and we have

$$
0 \leq \int_{\Omega} \mathcal{A}\left(x, D u_{j}\right) \cdot D \phi d x \rightarrow \int_{\Omega} \mathcal{A}(x, D u) \cdot D \phi d x \quad \text { as } j \rightarrow \infty .
$$

Since $E$ was arbitrary this concludes the proof.

\subsection{Harnack's Inequalities}

In order to get strong Harnack's inequality for $\mathcal{A}$-harmonic function and weak Harnack's inequality for $\mathcal{A}$-superharmonic functions we need related estimates proved for $\mathcal{A}$-subsolutions and $\mathcal{A}$-supersolutions. Having Lemma 3.7 we can specify results derived for quasiminizers in [25] to our case.

Proposition 4.7 (Corollary 3.6, [25]) For a locally bounded function $u \in W_{l o c}^{1, \varphi(\cdot)}(\Omega)$ being $\mathcal{A}$-subsolution in $\Omega$ there exist constants $R_{0}=R_{0}(n)>0$ and $C=$ $C$ (data, $n, R_{0}$, ess $\left.\sup _{B_{R_{0}}} u\right)>0$, such that

$$
\operatorname{ess}_{\sup } \operatorname{B}_{R / 2} u-k \leq C\left(\left(f_{B_{R}}(u-k)_{+}^{s} d x\right)^{\frac{1}{s}}+R\right)
$$

for all $R \in\left(0, R_{0}\right], s>0$ and $k \in \mathbb{R}$.

Proposition 4.8 (Theorem 4.3, [25]) For a nonnegative function $u \in W_{l o c}^{1, \varphi(\cdot)}(\Omega) \mathcal{A}$ supersolution in $\Omega$ there exist constants $R_{0}=R(n)>0, s_{0}=s_{0}($ data, $n)>0$ and $C=C($ data,$n)>0$, such that

$$
\left(f_{B_{R}} u^{s_{0}} d x\right)^{\frac{1}{s_{0}}} \leq C\left(\operatorname{essinf}_{B_{R / 2}} u+R\right)
$$

for all $R \in\left(0, R_{0}\right]$ provided $B_{3 R} \Subset \Omega$ and $\varrho_{\varphi(\cdot), B_{3 R}}(D u) \leq 1$.

Let us comment on the above result. For the application in [25] dependency of $s_{0}$ on other parameters is not important and so - not studied with attention. Actually, this theorem is not proven in detail in [25], but refers to standard arguments presented in [30, 32]. Their re-verification enables to find $s_{0}=s_{0}(\operatorname{dat} a, n)$. Let us note that after we completed our manuscript, an interesting study on the weak Harnack inequalities with an explicit exponent, holding for unbounded supersolutions, within our framework of generalized Orlicz spaces appeared, see [7].

Since $\mathcal{A}$-harmonic function is an $\mathcal{A}$-subsolution and and $\mathcal{A}$-supersolution at the same time (Lemma 3.4), by Propositions 4.7 and 4.8 we infer the full Harnack inequality.

Theorem 1 (Harnack's inequality for $\mathcal{A}$-harmonic functions) For a nonnegative $\mathcal{A}$ harmonic function $u \in W_{\text {loc }}^{1, \varphi(\cdot)}(\Omega)$ there exist constants $R_{0}=R(n)>0, s_{0}=$ $s_{0}($ data, $n)>0$ and $C=C\left(\right.$ data, $n, R_{0}$, $\left.\operatorname{ess~sup~}_{B_{R_{0}}} u\right)>0$, such that

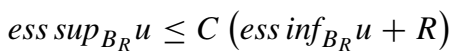

for all $R \in\left(0, R_{0}\right]$ provided $B_{3 R} \Subset \Omega$ and $\varrho_{\varphi(\cdot), B_{3 R}}(D u) \leq 1$. 


\subsection{Harnack's Principle for $\mathcal{A}$-Superharmonic Functions}

We are going to characterize the limit of nondecreasing sequence of $\mathcal{A}$-superharmonic functions and their gradients.

Theorem 2 (Harnack's Principle for $\mathcal{A}$-superharmonic functions) Suppose that $u_{i}, i=$ $1,2, \ldots$, are $\mathcal{A}$-superharmonic and finite a.e. in $\Omega$. If the sequence $\left\{u_{i}\right\}$ is nondecreasing then the limit function $u=\lim _{i \rightarrow \infty} u_{i}$ is $\mathcal{A}$-superharmonic or infinite in $\Omega$. Furthermore, if $u_{i}, i=1,2, \ldots$, are nonnegative, then up to a subsequence also $D u_{i} \rightarrow D u$ a.e. in $\{u<\infty\}$, where ' $D$ ' stands for the generalized gradient, $c f$. Eq. 6 .

Proof The proof is presented in three steps. We start with motivating that the limit function is either $\mathcal{A}$-superharmonic or $u \equiv \infty$, then we concentrate on gradients initially proving the claim for a priori globally bounded sequence $\left\{u_{i}\right\}$ and conclude by passing to the limit with the bound.

Step 1. Since $u_{i}$ are lower semicontinuous, so is $u$. The following fact holds: Given a compact set $K \Subset \Omega$, if $h \in C(K), \epsilon>0$ is a small fixed number, and $u>h-\epsilon$ on $K$, then, for $i$ sufficiently large, $u_{i}>h-\epsilon$. Indeed, let's argue by contradiction. Assume that for every $i$ there exists $x_{i} \in K$, such that

$$
u_{i}\left(x_{i}\right) \leq h\left(x_{i}\right)-\epsilon .
$$

Since $K$ is compact, we can assume that $x_{i} \rightarrow x_{o}$. Fix $l \in \mathbb{N}$. Then, for $i>l$ we have

$$
u_{l}\left(x_{i}\right) \leq u_{i}\left(x_{i}\right) \leq h\left(x_{i}\right)-\epsilon
$$

The right-hand side in the previous display tends with $i \rightarrow \infty$ to $h\left(x_{o}\right)-\epsilon$. Hence

$$
u_{l}\left(x_{o}\right) \leq \liminf _{i \rightarrow \infty} u_{l}\left(x_{i}\right) \leq h\left(x_{o}\right)-\epsilon
$$

Thus for every $l$ we have $u_{l}\left(x_{o}\right) \leq h\left(x_{o}\right)-\epsilon$, which implies $u\left(x_{o}\right) \leq h\left(x_{o}\right)-\epsilon$ which is in the contradiction with the fact that $u>h-\epsilon$ on $K$.

Using this fact we can prove that the limit function $u=\lim _{i \rightarrow \infty} u_{i}$ is $\mathcal{A}$-superharmonic unless $u \equiv \infty$. Choose an open $\Omega^{\prime} \Subset \Omega$ and $h \in C\left(\overline{\Omega^{\prime}}\right)$ an $\mathcal{A}$-harmonic function. Assume the inequality $u \geq h$ holds on $\partial \Omega^{\prime}$. It follows that for every $\epsilon>0$ on $\partial \Omega^{\prime}$ we have $u>h-\epsilon$ and, from the aforementioned fact, it follows that $u_{i}>h-\epsilon$ on $\partial \Omega^{\prime}$. Since all $u_{i}$ are $\mathcal{A}$-superharmonic, Proposition 4.1 yields that $u_{i} \geq h-\epsilon$ on $\Omega^{\prime}$. Therefore $u \geq h-\epsilon$ on $\Omega^{\prime}$. Since $\epsilon$ is arbitrary, we have $u \geq h$ on $\Omega^{\prime}$. Therefore the Comparison Principle from definition of $\mathcal{A}$-superharmonic holds unless $u \equiv \infty$ in $\Omega$. Finally, $u=\lim _{i \rightarrow \infty} u_{i}$ is $\mathcal{A}$-superharmonic unless $u \equiv \infty$.

Step 2. Assume $0 \leq u_{i} \leq k$ for all $i$ with $k>1$ and choose open sets $E \Subset G \Subset \Omega$. By Lemma 3.6 we get that

$$
\varrho_{\varphi(\cdot), G}\left(D u_{i}\right) \leq c k^{q}
$$

with $c=c($ data, $n)>0$ uniform with respect to $i$. Then, by doubling properties of $\varphi$, we infer that

$$
\left\|D u_{i}\right\|_{L^{\varphi(\cdot)}(G)} \leq c(\text { data, } n, k) .
$$

Consequently $\left\{u_{i}\right\}$ is bounded in $W^{1, \varphi(\cdot)}(G)$ and $u_{i} \rightarrow u$ weakly in $W^{1, \varphi(\cdot)}(G)$. Further, it has a non-relabelled subsequence converging a.e. in $G$ to $u \in W^{1, \varphi(\cdot)}(G)$. Let us show that

$$
D u_{j} \rightarrow D u \quad \text { a.e. in } E .
$$


We fix arbitrary $\varepsilon \in(0,1)$, denote

$$
J_{i}=\left\{x \in E:\left(\mathcal{A}\left(x, D u_{i}(x)\right)-\mathcal{A}(x, D u(x))\right) \cdot\left(D u_{i}(x)-D u(x)\right)>\varepsilon\right\}
$$

and estimate its measure. We have

$$
\begin{aligned}
\left|J_{i}\right| \leq & \left|J_{i} \cap\left\{\left|u_{i}-u\right| \geq \varepsilon^{2}\right\}\right| \\
& +\frac{1}{\varepsilon} \int_{J_{i} \cap\left\{\left|u_{i}-u\right|<\varepsilon^{2}\right\}}\left(\mathcal{A}\left(x, D u_{i}\right)-\mathcal{A}(x, D u)\right) \cdot\left(D u_{i}-D u\right) d x .
\end{aligned}
$$

Let $\eta \in C_{0}^{\infty}(G)$ be such that $\mathbb{1}_{E} \leq \eta \leq \mathbb{1}_{G}$. We define

$$
w_{1}^{i}=\min \left\{\left(u_{i}+\varepsilon^{2}-u\right)^{+}, 2 \varepsilon^{2}\right\} \quad \text { and } \quad w_{2}^{i}=\min \left\{\left(u+\varepsilon^{2}-u_{i}\right)^{+}, 2 \varepsilon^{2}\right\} .
$$

Then $w_{1}^{i} \eta$ and $w_{2}^{i} \eta$ are nonnegative functions from $W_{0}^{1, \varphi(\cdot)}(G)$ and can be used as test functions. Since $u$ and $u_{i}, i=1,2, \ldots$, are $\mathcal{A}$-supersolutions we already know that $u_{i} \rightarrow u$ weakly in $W^{1, \varphi(\cdot)}\left(E^{\prime}\right)$. By growth condition we can estimate like in Eq. 7 and by 14 we have

$$
\begin{aligned}
\int_{G \cap\left\{\left|u_{i}-u\right|<\varepsilon^{2}\right\}} \mathcal{A}(x, D u) \cdot\left(D u_{i}-D u\right) \eta d x & \leq \int_{G \cap\left\{\left|u_{i}-u\right|<\varepsilon^{2}\right\}} \mathcal{A}(x, D u) \cdot D \eta w_{1}^{i} d x \\
& \leq c \varepsilon^{2} \int_{G} \frac{\varphi(x,|D u|)}{|D u|}|D \eta| d x \\
& \leq c \varepsilon^{2}
\end{aligned}
$$

with $c>0$ independent of $i$ and $\varepsilon$. Analogously

$$
\int_{G \cap\left\{\left|u_{i}-u\right|<\varepsilon^{2}\right\}} \mathcal{A}\left(x, D u_{i}\right) \cdot\left(D u_{i}-D u\right) \eta d x \leq c \varepsilon^{2},
$$

Summing up the above observations we have

$$
\frac{1}{\varepsilon} \int_{J_{i} \cap\left\{\left|u_{i}-u\right|<\varepsilon^{2}\right\}}\left(\mathcal{A}\left(x, D u_{i}\right)-\mathcal{A}(x, D u)\right) \cdot\left(D u_{i}-D u\right) d x \leq c \varepsilon .
$$

The left-hand side is nonnegative by the monotonicity of the operator, so due to Eq. 16 we have

$$
\left|J_{i}\right| \leq\left|J_{i} \cap\left\{\left|u_{i}-u\right| \geq \varepsilon^{2}\right\}\right|+c \varepsilon
$$

with $c>0$ independent of $i$ and $\varepsilon$. By letting $\varepsilon \rightarrow 0$ we get that $\left|E_{j}\right| \rightarrow 0$. Because of the strict monotonicity of the operator, we infer (15). We can conclude the proof of this step by choosing a diagonal subsequence.

Step 3. Now we concentrate on the general case. For every $k=1,2, \ldots$ we select subsequences $\left\{u_{i}^{(k)}\right\}_{k}$ of $\left\{u_{i}\right\}$ and find an $\mathcal{A}$-superharmonic function $v_{k}$, such that $\left\{u_{i}^{(k+1)}\right\} \subset$ $\left\{u_{i}^{(k)}\right\}, T_{k}\left(u_{j}^{(k)}\right) \rightarrow v_{k}$ and $D\left(T_{k}\left(u_{j}^{(k)}\right)\right) \rightarrow D v_{k}$ a.e. in $\Omega$. We note that $v_{k}$ increases to a function, which is $\mathcal{A}$-harmonic or equivalently infinite. Additionally, $v_{k}=T_{k}(u)$. The diagonally chosen subsequence $\left\{u_{i}^{(i)}\right\}$ has all the desired properties.

We have the following consequence of the Comparison Principle and Theorem 2.

Corollary 4.9 (Harnack's Principle for $\mathcal{A}$-harmonic functions) Suppose that $u_{i}, i=$ $1,2, \ldots$, are $\mathcal{A}$-harmonic in $\Omega$. If the sequence $\left\{u_{i}\right\}$ is nondecreasing then the limit function $u=\lim _{i \rightarrow \infty} u_{i}$ is $\mathcal{A}$-harmonic or infinite in $\Omega$. 


\subsection{Poisson Modification}

The Poisson modification of an $\mathcal{A}$-superharmonic function in a regular set $E$ carries the idea of its local smoothing. A boundary point is called regular if at this point the boundary value of any Musielak-Orlicz-Sobolev function is attained not only in the Sobolev sense but also pointwise. A set is called regular if all of its boundary points are regular. See [25] for the result that if the complement of $\Omega$ is locally fat at $x_{0} \in \partial \Omega$ in the capacity sense, then $x_{0}$ is regular. Thereby of course polyhedra and balls are obviously regular.

Let us consider a function $u$, which is $\mathcal{A}$-superharmonic and finite a.e. in $\Omega$ and an open set $E \Subset \Omega$ with regular $\bar{E}$. We define

$$
u_{E}=\inf \left\{v: v \text { is } \mathcal{A} \text {-superharmonic in } E \text { and } \liminf _{y \rightarrow x} v(y) \geq u(x) \text { for each } x \in \partial \bar{E}\right\}
$$

and the Poisson modification of $u$ in $E$ by

$$
P(u, E)= \begin{cases}u & \text { in } \Omega \backslash E, \\ u_{E} & \text { in } E .\end{cases}
$$

Theorem 3 (Fundamental properties of the Poisson modification) If $u$ is $\mathcal{A}$-superharmonic and finite a.e. in $\Omega$, then its Poisson modification $P(u, E)$ is

(i) $\mathcal{A}$-superharmonic in $\Omega$,

(ii) $\mathcal{A}$-harmonic in $E$,

(iii) $\quad P(u, E) \leq u$ in $\Omega$.

Proof The fact that $P(u, E) \leq u$ in $\Omega$ results directly from the definition. By assumption $u$ is finite somewhere. Let us pick a nondecreasing sequence $\left\{\phi_{i}\right\} \subset C^{\infty}\left(\mathbb{R}^{n}\right)$ which converges to $u$ in $\bar{E}$. Let $h_{i}$ be the unique $\mathcal{A}$-harmonic function agreeing with $\phi_{i}$ on $\partial E$. The sequence $\left\{h_{i}\right\}$ is nondecreasing by the Comparison Principle from Proposition 4.1. Since $h_{i} \leq u$, by Harnack's Principle from Corollary 4.9 we infer that

$$
h:=\lim _{i \rightarrow \infty} h_{i}
$$

is $\mathcal{A}$-harmonic in $E$. Moreover, $h \leq u$ and thus $h$ is also finite somewhere. Since

$$
u(y)=\lim _{i \rightarrow \infty} \phi_{i}(y) \leq \liminf _{x \rightarrow y} h(x) \text { for } y \in \partial E,
$$

it follows that $P(u, E) \leq h$ in $E$. On the other hand, by the Comparison Principle (Proposition 4.1) we get that $h_{i} \leq P(u, E)$ in $E$ for every $i$. Therefore $\left.P(u, E)\right|_{E}=h$ is $\mathcal{A}$-harmonic in $E$. This reasoning also shows that $P(u, E)$ is lower semicontinuous and, by Lemma 4.3, it is also $\mathcal{A}$-superharmonic in $\Omega$.

\subsection{Minimum and Maximum Principles}

Before we prove the principles, we need to prove the following lemmas.

Lemma 4.10 If $u$ is $\mathcal{A}$-superharmonic and $u=0$ a.e. in $\Omega$, then $u \equiv 0$ in $\Omega$.

Proof It is enough to show that $u=0$ in a given ball $B \Subset \Omega$. By lower semicontinuity of $u$ infer that it is nonpositive. By Lemma 4.6 we get that $u \in W^{1, \varphi(\cdot)}(\Omega)$. Let $v=P(u, B)$ be the Poisson modification of $u$ in $B$. By Theorem 3 we have that $v$ is continuous in $B$ and 
$v \leq u \leq 0$. Therefore $v$ is an $\mathcal{A}$-supersolution in $\Omega$ and $(u-v) \in W_{0}^{1, \varphi(\cdot)}(\Omega)$. Moreover,

$$
c_{2}^{\mathcal{A}} \int_{\Omega} \varphi(x,|D v|) d x \leq \int_{\Omega} \mathcal{A}(x, D v) \cdot D v d x \leq \int_{\Omega} \mathcal{A}(x, D v) \cdot D u d x=0,
$$

where the last equality holds because $D u=0$ a.e. in $\Omega$. But then, we directly get that $D v=0$ and $v=0$ a.e. in $\Omega$. By continuity of $v$ in $B$ we get that $v=0$ everywhere in $B$. In the view of $v \leq u \leq 0$, we get that also $u \equiv 0$ in $\Omega$.

Lemma 4.11 If $u$ is $\mathcal{A}$-superharmonic and finite a.e. in $\Omega$, then for every $x \in \Omega$ it holds that $u(x)=\liminf _{y \rightarrow x} u(y)=$ ess $\liminf _{y \rightarrow x} u(y)$.

Proof We fix arbitrary $x \in \Omega$ and by lower semicontinuity $u(x) \leq \liminf _{y \rightarrow x} u(y) \leq$ ess $\liminf _{y \rightarrow x} u(y)=: a$. Let $\varepsilon \in(0, a)$ and $B=B(x, r) \subset \Omega$ be such that $u(y)>a-\varepsilon$ for a.e. $y \in B$. By Corollary 4.2 function $v=\min \{u-a+\varepsilon, 0\}$ is $\mathcal{A}$-superharmonic in $\Omega$ and $v=0$ a.e. in $B$. By Lemma $4.10 v \equiv 0$ in $\Omega$, but then $u(x) \geq a-\varepsilon$. Letting $\varepsilon \rightarrow 0$ we obtain that $u(x)=a$ and the claim is proven.

We define $\psi: \Omega \times[0, \infty) \rightarrow[0, \infty)$ is given by

$$
\psi(x, s)=\varphi(x, s) / s .
$$

Note that within our regime $s \mapsto \psi(\cdot, s)$ is strictly increasing, but not necessarily convex. Although in general $\psi$ does not generate the Musielak-Orlicz space, we still can define $\varrho_{\psi(\cdot), \Omega}$ by Eq. 4 useful in quantifying the uniform estimates for trucations in the following lemma.

Lemma 4.12 If for $u$ there exist $M, k_{0}>0$, such that for all $k>k_{0}$

$$
\varrho_{\varphi(\cdot), B}\left(D T_{k} u\right) \leq M k,
$$

then there exists a function $\zeta:[0,|B|] \rightarrow[0, \infty)$, such that $\lim _{s \rightarrow 0^{+}} \zeta(s)=0$ and for every measurable set $E \subset B$ it holds that for all $k>0$

$$
\varrho_{\psi(\cdot), E}\left(D T_{k} u\right) \leq \zeta(|E|) .
$$

Proof The result is classical when $p=q$, [34]. Therefore, we present the proof only for $p<q$. We start with observing that

$$
\begin{aligned}
|\{x \in B: \varphi(x,|D u|)>s\}| & \leq|\{x \in B:|u|>k\}|+|\{x \in B: \varphi(x,|D u|)>s,|u| \leq k\}| \\
& =I_{1}+I_{2} .
\end{aligned}
$$

Let us first estimate the volume of superlevel sets of $u$ using Tchebyszev inequality, Poincaré inequality, assumptions on the growth of $\varphi$, and Eq. 18. For all sufficiently large $k$ we have

$$
\begin{aligned}
I_{1} & =|\{x \in B:|u|>k\}| \leq \int_{B} \frac{\left|T_{k} u\right|^{p}}{k^{p}} d x \leq \frac{c}{k^{p}} \int_{B}\left|D T_{k} u\right|^{p} d x \\
& \leq \frac{c}{k^{p}} \int_{B} \varphi\left(x,\left|D T_{k} u\right|\right) d x=c k^{-p} \varrho_{\varphi(\cdot), B}\left(D T_{k} u\right) \leq c M k^{1-p} .
\end{aligned}
$$

Similarly by Tchebyszev inequality and Eq. 18 we can estimate also

$$
I_{2}=|\{x \in B: \varphi(x,|D u|)>s,|u| \leq k\}| \leq \frac{1}{s} \int_{\left\{\varphi\left(x, D T_{k} u\right)>s\right\}} \varphi\left(x, D T_{k} u\right) d x \leq M \frac{k}{s} .
$$


Altogether for all sufficiently large $s$ (i.e. $s>k_{0}^{p}$ ) we have that

$$
|\{x \in B: \varphi(x,|D u|)>s\}| \leq I_{1}+I_{2} \leq c s^{\frac{1-p}{p}} .
$$

Recall that due to Eq. 3 there exists $C>0$ uniform in $x$ such that $\psi(x, s) \geq$ $C \widetilde{\varphi}^{-1}(x, \varphi(x, s))$, so

$$
\begin{aligned}
|\{x \in B: \psi(x,|D u|)>s\}| & \leq\left|\left\{x \in B: C \widetilde{\varphi}^{-1}(x, \varphi(x,|D u|))>s\right\}\right| \\
& =|\{x \in B: \varphi(x,|D u|)>\widetilde{\varphi}(x, s / C)\}| \\
& \leq\left|\left\{x \in B: \varphi(x,|D u|)>(s / C)^{q^{\prime}}\right\}\right| \leq c s^{-\frac{q^{\prime}}{p^{\prime}}},
\end{aligned}
$$

for some $c>0$ independent of $x$. Since the case $q=p$ is trivial for these estimates, it suffices to consider $q>p$. Then $-\frac{q^{\prime}}{p^{\prime}}<-1$ and we get the uniform integrability of $\left\{\psi\left(\cdot,\left|D T_{k} u\right|\right)\right\}_{k}$, thus the claim follows.

Let us sum up the information on integrability of gradients of truncations of $\mathcal{A}$ superharmonic functions.

Remark 4.13 For a function $u$ being $\mathcal{A}$-superharmonic and finite a.e. in $\Omega$, by Lemma 4.6 we get that $\left\{T_{k} u\right\}$ is a sequence of $\mathcal{A}$-supersolutions in $\Omega$. Then Eq. 18 is satisfied because of the Caccioppoli estimate from Lemma 3.6. Having Lemma 4.12 we get that there exists $R_{0}>0$, such that for every $x \in \Omega$ and $B=B(x, R) \Subset \Omega$ with $R<R_{0}$ we have $\varrho_{\psi(\cdot), B}\left(D T_{k} u\right) \leq 1$ for all $k>0$ and in fact also $\varrho_{\psi(\cdot), B}(D u) \leq 1$ (where ' $D$ ' stands for the generalized gradient, cf. Eq. 6).

Lemma 4.14 For $u$ being a nonnegative function $\mathcal{A}$-superharmonic and finite a.e. in $\Omega$ there exist constants $R_{0}^{\mathcal{A}}=R_{0}^{\mathcal{A}}(n)>0, s_{0}=s_{0}($ data, $n)>0$ as in the weak Harnack inequality (Proposition 4.8), and $C=C($ data, $n)>0$, such that for every $k>1$ we have

$$
\left(f_{B_{R}}\left(T_{k} u\right)^{s_{0}} d x\right)^{\frac{1}{s_{0}}} \leq C\left(\inf _{B_{R / 2}}\left(T_{k} u\right)+R\right)
$$

for all $R \in\left(0, R_{0}^{\mathcal{A}}\right]$ provided $B_{3 R} \Subset \Omega$ and $\varrho_{\psi(\cdot), B_{3 R}}(D u) \leq 1$.

Proof The proof is based on Remark 4.13 and Proposition 4.8 that provides weak Harnack inequality for an $\mathcal{A}$-supersolution $v$ holding with constant $C=C$ (data, $n$ ) and for balls with radius $R<R_{0}(n)$ and so small that $\varrho_{\varphi(\cdot), B_{3 R_{0}}}(D v) \leq 1$.

The only explanation is required whenever $|D v| \geq 1$ a.e. in the considered ball. Then for every $k>1$ there exists $R_{1}(k)$ such that we get (19) for $T_{k} v$ over balls such that $R<\min \left\{R_{1}(k), R_{0}(n)\right\}$ and $\varrho_{\varphi(\cdot), B_{3 R_{1}(k)}}\left(D T_{k} v\right) \leq 1$. Of course, then there exists $R_{0}^{\mathcal{A}}(k) \in\left(0, R_{1}(k)\right)$, such that we have (19) for $R<\min \left\{R_{1}(k), R_{0}(n)\right\}$ and

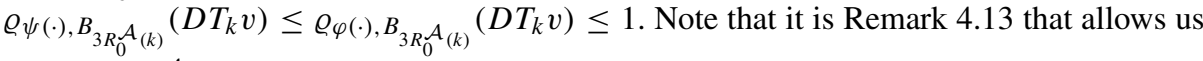
to choose $R_{0}^{\mathcal{A}}$ independently of $k$.

We are in a position to prove that an $\mathcal{A}$-harmonic function cannot attain its minimum nor maximum in a domain.

Theorem 4 (Strong Minimum Principle for $\mathcal{A}$-superharmonic functions) Suppose $u$ is $\mathcal{A}$ superharmonic and finite a.e. in connected set $\Omega$. If $u$ attains its minimum inside $\Omega$, then $u$ is a constant function. 
Proof We consider $v=\left(u-\inf _{\Omega} u\right)$, which by Corollary 4.2 is $\mathcal{A}$-superharmonic. Let $E=\{x \in \Omega: v(x)=0\}$, which by lower semicontinuity of $v$ (Lemma 4.11) is nonempty and relatively closed in $\Omega$. Having in hand Remark 4.13 we can choose $B=B(x, R) \subset$ $3 B \Subset \Omega$ with radius smaller than $R_{0}^{\mathcal{A}}$ from Lemma 4.14 and such that $\varrho_{\psi(\cdot), B_{3 R}}(D u) \leq 1$ where $\psi$ is as in Eq. 17. Therefore, in the rest of the proof we restrict ourselves to a ball $B$. By Corollary 4.2 functions $v$ and $T_{k} v$ are $\mathcal{A}$-superharmonic in $3 B$. Moreover, by Lemma 4.6 we infer that $\left\{T_{k} v\right\}$ is a sequence of $\mathcal{A}$-supersolutions integrable uniformly in the sense of Lemma 4.12. We take any $y \in B-$ a Lebesgue's point of $T_{k} v$ for every $k$ and choose $B^{\prime}=B^{\prime}\left(y, R^{\prime}\right) \Subset B$. Let us also fix arbitrary $k>0$. We have the weak Harnack inequality from Lemma 4.14 for $T_{k} v$ on $B^{\prime}$ yielding

$$
0 \leq\left(f_{B^{\prime}}\left(T_{k} v\right)^{s_{0}} d x\right)^{\frac{1}{s_{0}}} \leq C\left(\inf _{B^{\prime} / 2} T_{k} v+R^{\prime}\right)=C R^{\prime}
$$

with $s_{0}, C>0$ independent of $k$. Letting $R^{\prime} \rightarrow 0$ we get that $T_{k} v(y)=0$. Lebesgue's points of $T_{k} v$ for every $k$ are dense in $B$, we get that $T_{k} v \equiv 0$ a.e. in $B$. By arguments as in Lemma 4.10 we get that $T_{k} v \equiv 0$ in $B$, but then $B \subset E$ and $E$ has to be an open set. Since $\Omega$ is connected, $E$ is the only nonempty and relatively closed open set in $\Omega$, that is $E=\Omega$. Therefore $T_{k} v \equiv 0$ in $\Omega$. As $k>0$ was arbitrary $v=u-\inf _{\Omega} u \equiv 0$ in $\Omega$ as well.

The classical consequence of Strong Minimum Principle, we get its weaker form.

Corollary 4.15 (Minimum Principle for $\mathcal{A}$-superharmonic functions) Suppose $u$ is $\mathcal{A}$ superharmonic and finite a.e. in $\Omega$. If $E \Subset \Omega$ a connected open subset of $\Omega$, then

$$
\inf _{E} u=\inf _{\partial E} u .
$$

By the very definition of an $\mathcal{A}$-subharmonic function one gets the following direct consequence of the above fact.

Corollary 4.16 (Maximum Principle for $\mathcal{A}$-subharmonic functions) Suppose $u$ is $\mathcal{A}$ subharmonic and finite a.e. in $\Omega$. If $E \Subset \Omega$ a connected open subset of $\Omega$, then

$$
\sup _{E} u=\sup _{\partial E} u \text {. }
$$

Having Theorem 4 and Corollary 4.16, we infer that if $u$ is $\mathcal{A}$-harmonic in $\Omega$, then it attains its minimum and maximum on $\partial \Omega$. In other words $\mathcal{A}$-harmonic functions have the following Liouville-type property.

Corollary 4.17 (Liouville Theorem for $\mathcal{A}$-harmonic functions) If an $\mathcal{A}$-harmonic function attains its extremum inside a domain, then it is a constant function.

As a consequence of Minimum and Maximum Principles we derive one more inequality for $\mathcal{A}$-harmonic functions of the Harnack type.

Theorem 5 For a nonnegative function $u$ which is $\mathcal{A}$-harmonic in a connected set $\Omega$ there exist $R_{0}=R(n)>0$ and $C=C\left(\right.$ data, $n, R_{0}$, ess $\left.\sup _{B_{R_{0}}} u\right)>0$, such that

$$
\sup _{\partial B_{R}} u \leq C\left(\inf _{\partial B_{R}} u+R\right)
$$

for all $R \in\left(0, R_{0}\right]$ provided $B_{3 R} \Subset \Omega$ and $\varrho_{\psi(\cdot), B_{3 R}}(D u) \leq 1$, where $\psi$ is given by Eq. 17 . 
Proof It suffices to note that by Lemma 3.4 we can use Minimum Principle of Corollary 4.15 and Maximum Principle of Corollary 4.16. Then by Harnack inequality of Theorem 1 the proof is complete.

Corollary 4.18 Suppose $u$ is $\mathcal{A}$-harmonic in $B_{\frac{3}{2} R} \backslash B_{R}$, with $R<R_{0}$ from Theorem 5 , then exists $C=C\left(\right.$ data, $n, R_{0}$, ess $\left.\sup _{B_{R_{0}}} u\right)>0$, such that

$$
\sup _{\partial B_{\frac{4}{3} R}} u \leq C\left(\inf _{\partial B_{\frac{4}{3} R}} u+2 R\right) \text {. }
$$

Proof Fix $\varepsilon>0$ small enough for $B_{R} \Subset B_{\frac{4}{3} R-\varepsilon} \subset B_{\frac{4}{3} R+\varepsilon} \Subset B_{\frac{3}{2} R}$. Of course, then $u$ is $\mathcal{A}$-harmonic in $B_{\frac{4}{3} R+\varepsilon} \backslash B_{\frac{4}{3} R-\varepsilon}$. We cover the annulus with finite number of balls of equal radius as prescribed in the theorem and such that $\varrho_{\psi(\cdot), B}(D u) \leq 1$, which is possible due to Remark 4.13. Let us observe that due to the Harnack's inequality from Theorem 5 we have

$$
\begin{aligned}
\sup _{\partial B_{\frac{4}{3} R+\varepsilon} u} & \leq \sup _{\partial B_{\frac{4}{3} R+\varepsilon} \cup \partial B_{\frac{4}{3} R-\varepsilon}} u \leq C\left(\inf _{\partial B_{\frac{4}{3} R+\varepsilon} \cup B_{\frac{4}{3} R-\varepsilon}} u+\frac{4}{3} R+\varepsilon\right) \\
& \leq C\left(\inf _{\partial B_{\frac{4}{3} R+\varepsilon}} u+2 R\right) .
\end{aligned}
$$

Since $u$ is continuous in $B_{\frac{3}{2} R} \backslash B_{R}$, passing with $\varepsilon \rightarrow 0$ we get the claim.

Acknowledgements I. Chlebicka is supported by NCN grant no. 2019/34/E/ST1/00120. A. ZatorskaGoldstein is supported by NCN grant no. 2019/33/B/ST1/00535.

Open Access This article is licensed under a Creative Commons Attribution 4.0 International License, which permits use, sharing, adaptation, distribution and reproduction in any medium or format, as long as you give appropriate credit to the original author(s) and the source, provide a link to the Creative Commons licence, and indicate if changes were made. The images or other third party material in this article are included in the article's Creative Commons licence, unless indicated otherwise in a credit line to the material. If material is not included in the article's Creative Commons licence and your intended use is not permitted by statutory regulation or exceeds the permitted use, you will need to obtain permission directly from the copyright holder. To view a copy of this licence, visit http://creativecommons.org/licenses/by/4.0/.

\section{References}

1. Alberico, A., Chlebicka, I., Cianchi, A., Zatorska-Goldstein, A.: Fully anisotropic elliptic problems with minimally integrable data. Calc. Var. Partial Differential Equations 58, 186 (2019)

2. Adams, D.R., Hedberg, L.I.: Function Spaces and Potential Theory, Volume 314 of Grundlehren Der Mathematischen Wissenschaften [Fundamental Principles of Mathematical Sciences]. Springer, Berlin (1996)

3. Ahmida, Y., Chlebicka, I., Gwiazda, P., Youssfi, A.: Gossez's approximation theorems in MusielakOrlicz-Sobolev spaces. J. Functional Analysis 275(9), 2538-2571 (2018)

4. Alkhutov, Y.A.: The Harnack inequality and the Hölder property of solutions of nonlinear elliptic equations with a nonstandard growth condition. (Russian. Russian summary) Differ. Uravn. 33(12), 1651-1660 (1997). Translation in Differential Equations 33(12), 1653-1663 (1998)

5. Baroni, P., Colombo, M., Mingione, G.: Nonautonomous functionals, borderline cases and related function classes. Algebra i Analiz 27(3), 6-50 (2015). Translation in St. Petersburg Math. J. 27(3), 347-379 (2016) 
6. Bénilan, P., Boccardo, L., Gallouët, T., Gariepy, R., Pierre, M., Vázquez, J.-L.: An $l^{1}$-theory of existence and uniqueness of solutions of nonlinear elliptic equations. Ann. Scuola Norm. Sup. Pisa Cl. Sci. (4) 22(2), 241-273 (1995)

7. Benyaiche, A., Harjulehto, P., Hästö, P., Karppinen, A.: The weak Harnack inequality for unbounded supersolutions of equations with generalized Orlicz growth. J. Differential Equations 275, 790-814 (2021)

8. Björn, A., Björn, J.: Nonlinear Potential Theory on Metric Spaces EMS Tracts in Mathematics, vol. 17. European Mathematical Society (EMS), Zürich (2011)

9. Byun, S.-S., Cho, Y., Park, J.-T.: Nonlinear gradient estimates for elliptic double obstacle problems with measure data, arXiv:1912.04073 (2019)

10. Challal, S., Lyaghfouri, A., Rodrigues, J.F.: On the A-obstacle problem and the Hausdorff measure of its free boundary. Ann. Mat. Pura Appl. (4) 191(1), 113-165 (2012)

11. Chlebicka, I.: A pocket guide to nonlinear differential equations in musielak-Orlicz spaces. Nonl. Analysis 175, 1-27 (2018)

12. Chlebicka, I.: Gradient estimates for problems with Orlicz growth. Nonl. Analysis 194, 111364 (2020)

13. Chlebicka, I.: Measure data elliptic problems with generalized Orlicz growth, arXiv:2008.02495 (2020)

14. Chlebicka, I.: Regularizing effect of the lower-order terms in elliptic problems with Orlicz growth. Israel J. Math. 236(2), 967-1000 (2020)

15. Chlebicka, I., De Filippis, C.: Removable sets in non-uniformly elliptic problems. Ann. Mat Pura Appl. (4) 199(2), 619-649 (2020)

16. Chlebicka, I., Karppinen, A.: Removable sets in elliptic equations with Musielak-Orlicz growth. J. Math. Anal. Appl. https://doi.org/10.1016/j.jmaa.2020.124073 (2020)

17. Chlebicka, I., Giannetti, F., Zatorska-Goldstein, A.: Elliptic problems in the Orlicz setting without growth restrictions with measure or $L^{1}$ data. J. Math. Anal Appl. 479(1), 185-213 (2019)

18. Chlebicka, I., Giannetti, F., Zatorska-Goldstein, A.: Wolff potentials and local behaviour of solutions to measure data elliptic problems with Orlicz growth. arXiv:2006.02172 (2020)

19. Chlebicka, I., Gwiazda, P., Świerczewska-Gwiazda, A., Wróblewska-Kamińska, A.: Partial Differential Equations in anisotropic musielak-Orlicz spaces, preprint (2019)

20. Cianchi, A., Maz'ya, V.: Quasilinear elliptic problems with general growth and merely integrable, or measure, data. Nonlinear Anal. 164, 189-215 (2017)

21. Colombo, M., Mingione, G.: Regularity for double phase variational problems, arch. Rational Mech. Anal. 215, 443-496 (2015)

22. Cruz-Uribe, D., Hästö, P.: Extrapolation and interpolation in generalized Orlicz spaces. Trans. Amer. Math. Soc. 370(6), 4323-4349 (2018)

23. Di Marco, T., Marcellini, P.: A-priori gradient bound for elliptic systems under either slow or fast growth conditions. Calc. Var. Partial Differential Equations 59, 120 (2020)

24. Fang, Y., Zhang, C.: Equivalence between distributional and viscosity solutions for the double-phase equation. Adv. Calc. Var. https://doi.org/10.1515/acv-2020-0059

25. Harjulehto, P., Hästö, P.: Boundary regularity under generalized growth conditions. Z. Anal. Anwendungen 38(1), 73-96 (2020)

26. Harjulehto, P., Hästö, P.: Orlicz Spaces and Generalized Spaces, vol. 2236 of Lecture Notes in Mathematics Orlicz. Springer, Cham (2019)

27. Harjulehto, P., Hästö, P., Koskenoja, M., Lukkari, T., Marola, N.: An obstacle problem and superharmonic functions with nonstandard growth. Nonlinear Anal. 67(12), 3424-3440 (2007)

28. Harjulehto, P., Hästö, P., Latvala, V., Toivanen, O.: The strong minimum principle for quasisuperminimizers of non-standard growth. Ann. Inst. H. Poincaré, Anal. Non Linéaire 28(5), 731-742 (2011)

29. Harjulehto, P., Hästö, P., Lee, M.: Hölder continuity of quasiminimizers and $\omega$-minimizers of functionals with generalized Orlicz growth. Ann. Scuola Norm. Sup. Pisa Cl. Sci (4). https://doi.org/10.2422/2036-2145.201908_015 (2020)

30. Harjulehto, P., Hästö, P., Toivanen, O.: Hölder regularity of quasiminimizers under generalized growth conditions. Calc. Var. Partial Differential Equations 56(2), 56:22 (2017)

31. Harjulehto, P., Juusti, J.: The Kellogg property under generalized growth conditions, preprint (2020)

32. Harjulehto, P., Kuusi, T., Lukkari, T., Marola, N., Parviainen, M.: Harnack's inequality for quasiminimizers with nonstandard growth conditions. J. Math. Anal. Appl. 344, 504-520 (2008)

33. Havin, M., Maz'ya, V.G.: A nonlinear analogue of the Newtonian potential, and metric properties of $(p, l)$-capacity. Dokl. Akad. Nauk SSSR 194, 770-773 (1970)

34. Heinonen, J., Kilpeläinen, T., Martio, O.: Nonlinear Potential Theory of Degenerate Elliptic Equations. Unabridged Republication of the 1993 Original. Dover Publications, Inc., Mineola (2006) 
35. Hedberg, L.I., Wolff, T.H.: Thin sets in nonlinear potential theory. Ann. Inst Fourier (Grenoble) 33(4), 161-187 (1983)

36. Karppinen, A.: Global continuity and higher integrability of a minimizer of an obstacle under generalized Orlicz growth conditions. Manuscripta Math. 164(1-2), 67-94 (2021)

37. Karppinen, A., Lee, M.: Hölder continuity of the minimizer of an obstacle problem with generalized Orlicz growth, preprint (2019)

38. Kilpeläinen, T., Malý, J.: Degenerate elliptic equations with measure data and nonlinear potentials. Ann. Scuola Norm. Sup. Pisa Cl. Sci. (4) 19(4), 591-613 (1992)

39. Kilpeläinen, T., Kuusi, T., Tuhola-Kujanpää, A.: Superharmonic functions are locally renormalized solutions. Ann. Inst. H. Poincaré Anal. Non Linéaire 28(6), 775-795 (2011)

40. Kilpeläinen, T., Zhong, X.: Removable set for continuous solutions of quasilinear elliptic equations. Proc. Amer. Math. Soc. 130(6), 1681-1688 (2000)

41. Korte, R., Kuusi, T.: A note on the Wolff potential estimate for solutions to elliptic equations involving measures. Adv. Calc. Var. 3, 99-113 (2010)

42. Kuusi, T., Mingione, G.: Guide to nonlinear potential estimates. Bull. Math. Sci. 4(1), 1-82 (2014)

43. Kuusi, T., Mingione, G.: Linear potentials in nonlinear potential theory. Arch. Ration. Mech Anal. 207, 215-246 (2013)

44. Latvala, V., Lukkari, T., Toivanen, O.: The fundamental convergence theorem for $p(\cdot)$-superharmonic functions. Potential Anal. 35(4), 329-351 (2011)

45. Lieberman, G.M.: The natural generalization of the natural conditions of Ladyzhenskaya and Ural'tseva for elliptic equations. Comm. Partial Differential Equations 16(2-3), 311-361 (1991)

46. Malý, J.: Wolff potential estimates of superminimizers of Orlicz type Dirichlet integrals. Manuscripta Math. 110(4), 513-525 (2003)

47. Marcellini, P.: A variational approach to parabolic equations under general and $p, q$-growth conditions. Nonl. Analysis 194, 111456 (2020)

48. Mikkonen, P.: On the Wolff potential and quasilinear elliptic equations involving measures. Ann. Acad. Sci. Fenn. Math. Diss. 104, 71pp (1996)

49. Mingione, G.: Gradient estimates below the duality exponent. Math. Ann. 346(3), 571-627 (2010)

50. Rădulescu, V.: Isotropic and anisotropic double-phase problems: old and new. Opuscula Math. 39(2), 259-279 (2019)

51. Rodrigues, J.F., Teymurazyan, R.: On the two obstacles problem in Orlicz-Sobolev spaces and applications. Complex Var. Elliptic Equ. 56(7-9), 769-787 (2011)

52. Trudinger, N.S., Wang, X.-J.: On the weak continuity of elliptic operators and applications to potential theory. Amer. J. Math. 124, 369-410 (2002)

53. Turesson, B.O.: Nonlinear Potential Theory and Weighted Sobolev Spaces Lecture Notes in Mathematics, vol. 1736. Springer, Berlin (2000)

Publisher's Note Springer Nature remains neutral with regard to jurisdictional claims in published maps and institutional affiliations. 